\title{
A residual range cell migration correction algorithm for bistatic forward-looking SAR
}

\author{
Wei Pu*, Yulin Huang, Junjie Wu, Jianyu Yang and Wenchao Li
}

\begin{abstract}
For bistatic forward-looking synthetic aperture radar (BFSAR), images are often blurred by uncompensated radar motion errors. To get refocused images, autofocus is a useful postprocessing technique. However, a severe drawback of the autofocus algorithms is that they are only capable of removing one-dimensional azimuth phase errors. In BFSAR, motion errors and approximations of imaging algorithms introduce residual range cell migration (RCM) on BFSAR data as well. When residual RCM is within a range resolution cell, it can be neglected. However, the residual migration, which exceeds a range cell, is increasingly encountered as resolution becomes finer and finer. A novel residual RCM correction method is proposed in this paper. By fitting the low-frequency phase difference of adjacent azimuth cells, residual RCM of each azimuth cell can be corrected precisely and effectively. Simulations and real data experiments are carried out to validate the effectiveness of the proposed method.
\end{abstract}

Keywords: Bistatic forward-looking synthetic aperture radar (BFSAR), Residual range cell migration (RCM), Low-frequency fitting

\section{Introduction}

Forward-looking imaging is highly desirable in some applications, such as airplane navigation and landing. Due to the ability to obtain high-resolution image of the forward-looking terrain, bistatic synthetic aperture radar (SAR) is drawing more and more attention in recent years. However, the moving platforms introduce relative motion between radars and observed scene, which induces range cell migration (RCM) to bistatic forward-looking SAR data. The procedure of RCM correction is essential for the frequency domain imaging algorithms.

Usually, RCM cannot be corrected completely in practical application. The reason is that there are some residual components, namely residual RCM. And the residual RCM is introduced by motion errors [1]. In the presumption that residual RCM is within a range resolution cell, residual RCM can be neglected [2, 3], and motion errors can be compensated by autofocus methods completely. Generally speaking, residual RCM is relatively small and can be neglected in monostatic SAR, while the unique characteristics of BFSAR makes the residual RCM

*Correspondence: pwuestc@163.com

Department of Electronic Engineering, University of Electronic Science and

Technology of China, 2006 Xiyuan Road, Gaoxin Western District, 611731

Chengdu, China exceeding range resolution cell inevitable. On the one hand, the separated platforms of BFSAR induce motion errors much larger than the errors in monostatic SAR raw data. On the other hand, the higher order terms of range migration are always neglected in BFSAR imaging algorithms. However, the impacts of these higher order terms become serious when the squint angle gets larger and the resolution gets higher. In this situation, residual RCM correction becomes a necessary procedure for BFSAR imaging. In principle, it is possible to compute the residual RCM from orbit and attitude data provided by an ancillary instrument such as inertial measurement units (IMU) and global positioning system (GPS). Nevertheless, measurement uncertainties on the data would limit the accuracy, and the data remains unknown for some unmanned aerial vehicles without ancillary instrument. Thus, residual RCM correction based on SAR data is indispensable.

To correct residual RCM based on SAR data, three alternative strategies are available. (1) Estimate the azimuth phase error term firstly, and then calculate the residual RCM from the estimated azimuth phase error [4]. In [4], the range compressed data is processed to a new coarser range resolution so that the presumption of autofocus is met. Therefore, the azimuth phase errors can 
be obtained using autofocus method from the new range compressed data and then the residual RCM can be calculated and compensated. However, as the azimuth phase errors are estimated in coarse resolution, the estimation precision of azimuth phase errors and residual RCM cannot satisfy the demands of high-resolution BFSAR. (2) Estimate residual RCM by making use of the relationship between azimuth phase errors and residual RCM. Mao et al. [3] deduces an accurate analytical relationship between azimuth phase errors and residual RCM in spotlight SAR. Using this relationship, a 2-D autofocus method, which can compensate the azimuth phase errors and residual RCM simultaneously, is proposed. Nevertheless, this relationship is valid just in the case of spotlight SAR data, so that applicability of this method is limited. (3) Estimate the residual RCM independently. The residual RCM correction method proposed in [5] is based on range alignment algorithm which is utilized in translational motion compensation on inverse SAR (ISAR) [6, 7]. However, parametric model of the range displacement in [6] restricts the estimation accuracy, and the interpolation procedure in [7] requires exhaustive computation.

In [8], a novel residual RCM correction algorithm based on low-frequency fitting is proposed for monostatic SAR to correct the motion-induced errors. In this paper, this residual RCM correction algorithm is greatly improved to cope with BFSAR data. By estimating the residual RCM utilizing the least-squares method in the low-frequency area and compensating the displacement in frequency domain, a sub-pixel level correction result can be achieved in this proposed method. Compared with previous works on residual RCM correction of SAR data, the proposed method can realize residual RCM correction with accuracy of sub-pixel level but requiring neither interpolation nor parametric model. Compared with [8], the novelty of the proposed method in this paper includes two parts. Firstly, a phase difference denoising procedure is added to make the proposed method more robust and can be adapted to the practical condition. Secondly, the one isolated dominant target assumption is not indeed needed for the proposed method, which makes the proposed method can be widely used in most practical condition. Furthermore, the range curvature term and even higher order terms, which is the unique problem in BFSAR, also can be solved by the proposed method. Simulation and real BFSAR data-processing results are presented to verify the effectiveness of the proposed method.

\section{Problem formulation}

The geometric model in Fig. 1 provides the basis for BFSAR imaging. In the ideal condition, the transmitter and receiver are moving along parallel tracks with equal velocity. The squint angles $\phi_{T}$ and $\phi_{R}$ and initial ranges $R_{T \text { cen }}$ and $R_{R \text { cen }}$ shown in Fig. 1 are measured at the

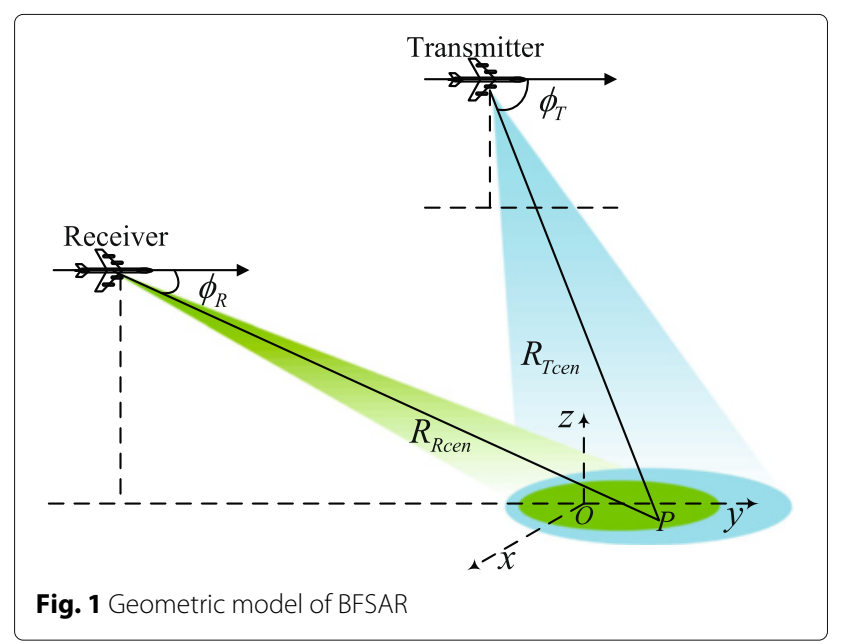

composite beam center crossing time of the reference target $P$.

Assume that linear frequency modulated (LFM) pulses are transmitted by the radar. The demodulated signal from the reference target in the presence of motion errors can be adequately described by

$$
\begin{aligned}
s(\tau, t)= & \operatorname{rect}\left[\frac{\tau-\bar{R}(t) / c}{T_{r}}\right] \operatorname{rect}\left(\frac{t}{T_{a}}\right) \\
& \times \exp \left\{-j \pi K_{r}\left[\tau-\frac{\bar{R}(t)}{c}\right]^{2}\right\} \\
& \times \exp \left\{-j 2 \pi \frac{\bar{R}(t)}{\lambda}\right\}
\end{aligned}
$$

where $K_{r}$ is the transmitted chirp rate, $T_{r}$ is the timewidth of the LFM pulse, and $T_{a}$ is the synthetic aperture time. The range time is given by $\tau$, and $t$ denotes the crossrange time, $\lambda$ is the wavelength, and $c$ is the speed of propagation.

In (1), $\bar{R}(t)$ is the instantaneous two-way range of reference target $P$ in the presence of motion errors. $\bar{R}(t)$ can be formulated as

$$
\bar{R}(t)=R(t)+\delta R(t)
$$

where $\delta R(t)$ denotes the instantaneous range displacement induced by motion errors, and $R(t)$ represents the nominal instantaneous two-way range.

$$
\begin{aligned}
R(t)= & \sqrt{R_{T \mathrm{cen}}^{2}+(V t)^{2}-2 R_{T \operatorname{cen}} V t \cos \phi_{T}} \\
& +\sqrt{R_{R \operatorname{cen}}^{2}+(V t)^{2}-2 R_{R \operatorname{cen}} V t \cos \phi_{R}}
\end{aligned}
$$


Expand (3) at $t=0$ to its Taylor series and $R(t)$ can be rewritten as

$$
\begin{aligned}
R(t)= & R_{T \text { cen }}+R_{R \text { cen }} \\
& -\left(V \cos \phi_{R}+V \cos \phi_{T}\right) t \\
& +\left(\frac{V^{2} \sin ^{2} \phi_{R}}{2 R_{R \operatorname{cen}}}+\frac{V^{2} \sin ^{2} \phi_{T}}{2 R_{T \text { cen }}}\right) t^{2}+\cdots
\end{aligned}
$$

The linear and quadratic terms in (4) are called the range walk and range curvature, respectively. In BFSAR, the total range cell migration (RCM) is dominated by the range walk component [9]. Usually, linear RCM correction (LRCMC) procedures in azimuthal time domain like squint minimization [10] and keystone transform [11] are utilized to correct the range walk. After range compression and LRCMC, a coarse range focusing signal $s^{\prime}(\tau, t)$ is obtained.

$$
\begin{aligned}
s^{\prime}(\tau, t)= & \sin c\left[\tau-\frac{R_{T \text { cen }}+R_{R \text { cen }}}{c}-\frac{\Delta R(t)}{c}\right] \\
& \operatorname{rect}\left(\frac{t}{T_{a}}\right) \exp \left[-j \frac{2 \pi}{\lambda} \bar{R}(t)\right]
\end{aligned}
$$

where $\Delta R(t)$ in the range profile is

$$
\Delta R(t)=\delta R(t)+\left(\frac{V^{2} \sin ^{2} \phi_{R}}{2 R_{R \operatorname{cen}}}+\frac{V^{2} \sin ^{2} \phi_{T}}{2 R_{T \text { cen }}}\right) t^{2}+\cdots
$$

In (5), the displacement $\Delta R(t) / c$ in the range profile is residual RCM. To illustrate the influence of residual RCM, the simple sketch map of residual RCM is shown in Fig. 2.
In Fig. 2, the solid line denotes the migration trajectory of a prominent scatter after range compression and range cell migration.

The nonlinear migration trajectory in Fig. 2 is introduced by residual RCM. When the residual RCM exceeds a range resolution, 2-D defocus will emerge in the final image. The reason is that the energy of target scatter diffuses in several range cells and induces image defocus in range direction. Besides, the general presumption of autofocus is not valid, and there is considerable challenge for autofocus methods. When the autofocus procedures are performed in the azimuth direction, the azimuth signal is assumed to be a LMF signal. While in the condition that the residual RCM exceeds a range resolution, the energy of one target scatter diffuses in several range cells. Thus, the azimuth signal in a given range bin is not a complete LFM signal any more. Autofocus methods can not be correctly utilized as for a incomplete LFM signal. Hence, autofocus techniques are not effective if the residual errors are larger than range cell. And therefore, the image results defocus in azimuth direction as well.

Therefore, in order to obtain high-resolution BFSAR image, residual RCM correction is essential.

\section{Residual RCM correction}

In this section, a novel residual RCM correction method is proposed. Residual RCM of each azimuth cell is corrected one by one. Here, the residual RCM correction method for one azimuth cell is presented in the rest of this section.

\subsection{Row correlation analysis}

Let $f_{m}[n]$ and $f_{m+1}[n]$ denote the adjacent azimuth cells of BFSAR data as shown in Fig. 2, where $m=1,2, \cdots, M$

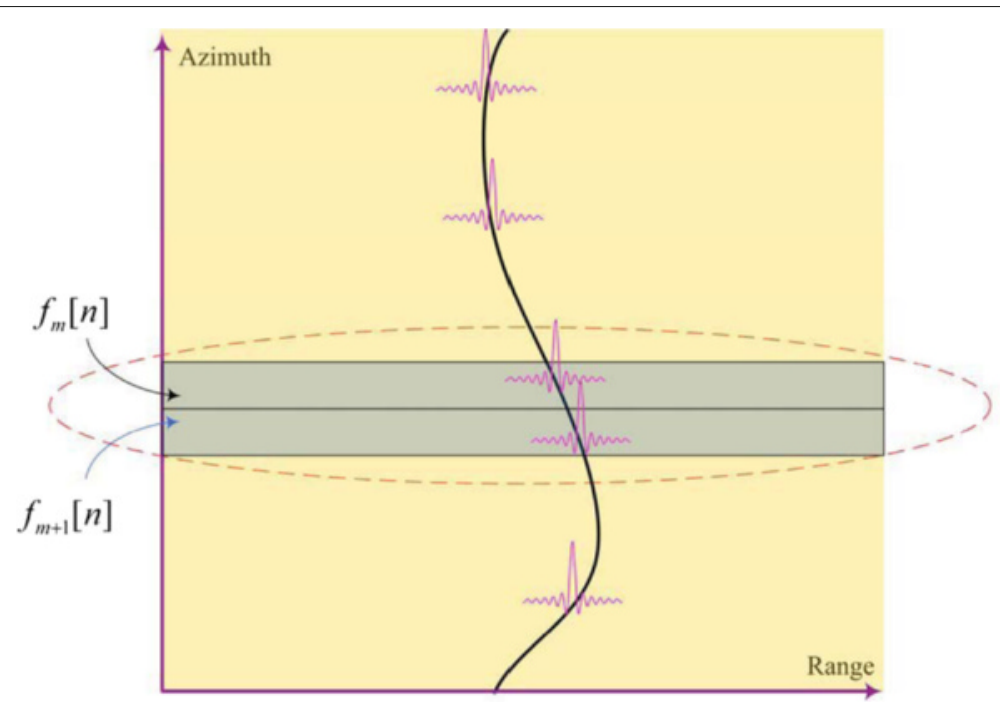

Fig. 2 Illustration of residual RCM 
indexes the transmitted pulses and $n=1,2, \cdots, N$ stands for the samples taken from each pulses, $M$ is the azimuth sample number, and $N$ is the range sample number.

According to (5), $f_{m}[n]$ and $f_{m+1}[n]$ can be written as

$$
\begin{gathered}
f_{m}[n]=A \sin c\left(n-\frac{R_{0}}{c}-\frac{\Delta R_{m}}{c}\right) \exp \\
f_{m+1}[n]=A \sin c\left(n-\frac{R_{0}}{c}-\frac{\Delta R_{m+1}}{c}\right) \\
\exp \left(-j \frac{2 \pi \bar{R}_{m+1}}{\lambda}\right)
\end{gathered}
$$

where $\bar{R}_{m}$ and $\Delta R_{m}$ denote the slant range and range displacement measured at the $m$ th transmitted pulse, respectively; $\bar{R}_{m+1}$ and $\Delta R_{m+1}$ denote the slant range and range displacement measured at the $(m+1)$ th transmitted pulse, respectively.

From (7) and (8), the relationship between the adjacent azimuth cells $f_{m}[n]$ and $f_{m+1}[n]$ can be deduced.

$$
\left|f_{m+1}[n]\right|=\left|f_{m}\left[n-n_{m+1}\right]\right|
$$

where $n_{m+1}$ denotes the displacement between $\left|f_{m}[n]\right|$ and $\left|f_{m+1}[n]\right|$.

$$
n_{m+1}=\frac{\Delta R_{m+1}-\Delta R_{m}}{c}
$$

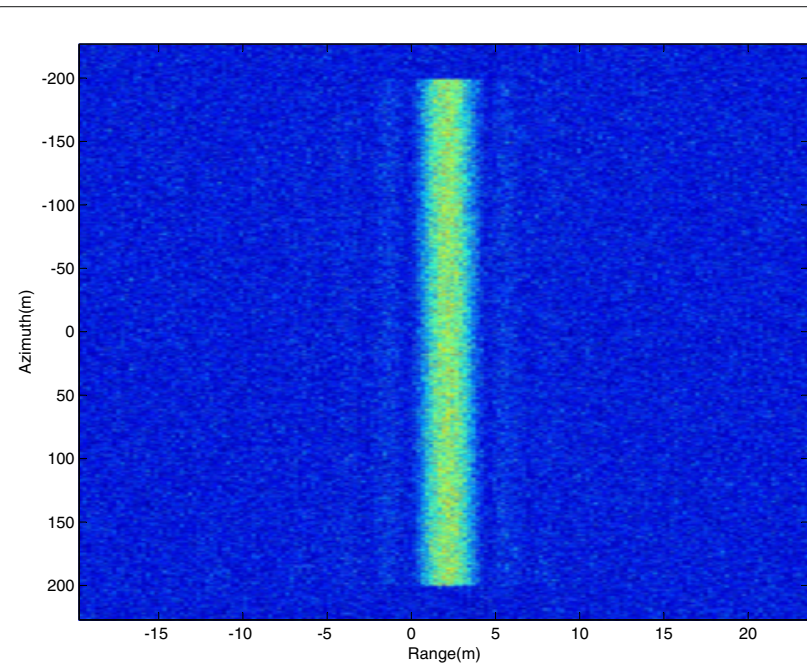

(a)
That is to say, apart from the displacement $n_{m+1}$, two adjacent rows of the data can be considered as approximately equal.

The similarity of adjacent rows can be measured by the correlation value $(\mathrm{CV})$, which is defined as:

$$
r=\frac{\sum_{i}\left(g_{m+1}[n]-\bar{g}_{m+1}\right)\left(g_{m}[n]-\bar{g}_{m}\right)}{\sqrt{\left[\sum_{n}\left(g_{m+1}[n]-\bar{g}_{m+1}\right)^{2}\right]\left[\sum_{n}\left(g_{m}[n]-\bar{g}_{m}\right)^{2}\right]}}
$$

where $r$ is the correlation value of adjacent rows,

$$
\begin{aligned}
& g_{m+1}[n]=\left|f_{m+1}[n]\right| \\
& g_{m}[n]=\left|f_{m}[n]\right|
\end{aligned}
$$

In this article, it is assumed that the adjacent rows of data can be regarded as approximately equal if the average $\mathrm{CV}$ is larger than 0.85 . Fortunately, the range focused data of natural scenes collected by BFSAR system can satisfy this condition. Figure 3 a presents the simulated range focused data with one target, and Fig. 3b depicts the correlation function curve of the range focused data. White Gauss noise with $\mathrm{SNR}=5 \mathrm{~dB}$ is added into the simulated data. Simulation parameters are listed in Table 1. It can be seen that the CVs meet the requirements completely.

Taking the real data of BFSAR as an example, and calculating the correlation value of 10,000 group adjacent rows, respectively, we can get the correlation function curve in Fig. 4b. We carry out an airborne BFSAR experiment in 2012 [12]. The actual data comes from the experiment. It

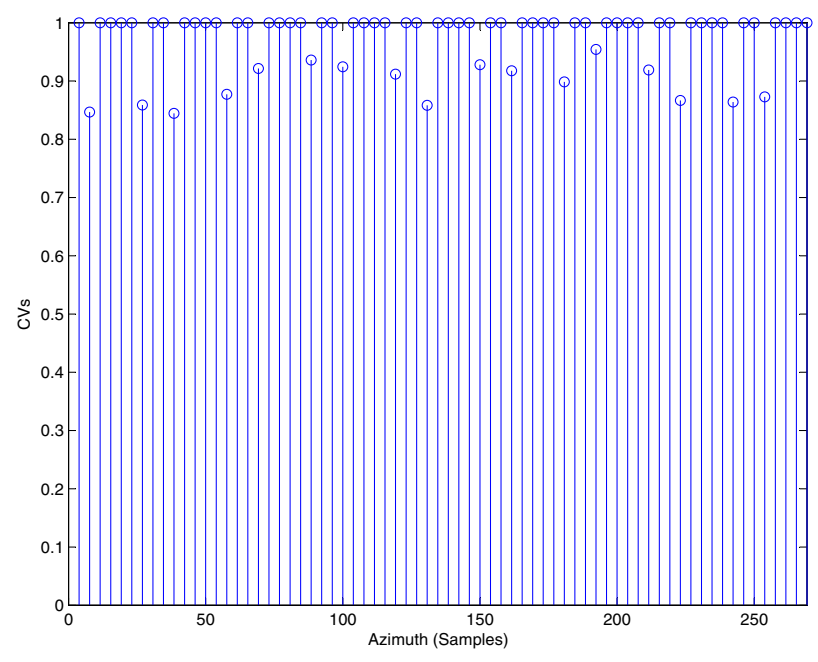

(b)

Fig. 3 a Range focused signal of the simulation data. $\mathbf{b}$ CV between adjacent rows 
Table 1 Simulation parameters

\begin{tabular}{ll}
\hline Parameter & Value \\
\hline Carrier frequency & $10 \mathrm{GHz}$ \\
Band width & $400 \mathrm{MHz}$ \\
Synthetic aperture time & $5 \mathrm{~s}$ \\
Nominal Radar platform velocity & $100 \mathrm{~m} / \mathrm{s}$ \\
Pulse repetition frequency & $600 \mathrm{~Hz}$ \\
Coordinates of the transmitter & $(1,0.6,0.8) \mathrm{km}$ \\
Coordinates of the receiver & $(0,1.2,0.7) \mathrm{km}$ \\
Size of mean filter window & 3 \\
\hline
\end{tabular}

is shown in Fig. $4 \mathrm{~b}$ that due to the continuity of the scene, the CVs of adjacent rows are all above 0.87 , i.e., the adjacent rows of data can be regarded as approximately equal. It can be seen that the CVs meet the requirements as for the BFSAR data as well.

Hence, it is reasonable to regard the two adjacent rows of the data as approximately equal, and this feature provides the theoretical basis for the proposed algorithm.

\subsection{Phase difference extraction}

According to (7), (8), and (10), we can write

$$
\begin{aligned}
F_{m+1}(\omega)= & F_{m}(\omega) \\
& \exp \left[-j \omega n_{m+1}-j \frac{2 \pi\left(\bar{R}_{m+1}-\bar{R}_{m}\right)}{\lambda}\right]
\end{aligned}
$$

where $F_{m+1}(\omega)$ and $F_{m}(\omega)$ are the frequency spectra of $f_{m+1}[n]$ and $f_{m}[n]$, respectively. Then, phase difference between $F_{m+1}(\omega)$ and $F_{m}(\omega)$ can be calculated by Eq. (14).

$$
\Phi(\omega)=-n_{m+1} \omega-\frac{2 \pi\left(\bar{R}_{m+1}-\bar{R}_{m}\right)}{\lambda}
$$

where $\Phi(\omega) \in(-\pi, \pi]$ denotes the phase difference between $F_{m+1}(\omega)$ and $F_{m}(\omega)$. As known to all, the phase is limited to the interval of $(-\pi, \pi]$ and leads to the transition of a phase difference from $-\pi$ to $\pi$. In order to get a continuous phase difference curve, it is necessary to unwrap the phase spectrum while limited SNR of a BFSAR data can result in a great difference between adjacent rows of data. Hence, there will be a large local jitter in the phase difference curve, which will lead to failure of phase unwrapping. As a result, the number of fitting points to a linear function would decrease. Therefore, the phase difference curve should be denoised and smoothed before unwrapping.

\subsection{Phase difference denoising}

A median filter or a mean filter can not be simply applied into the denoising of phase, because they would seriously destroy the transition property in the phase difference spectrum. Let $\Phi[n]$ denote the discrete version of the $\Phi(\omega)$. Consider the phase sequence

$$
\begin{gathered}
a[n]=\cos (\Phi[n]) \\
b[n]=\sin (\Phi[n])
\end{gathered}
$$

The formulas mentioned above make the original phase $\Phi[n]$ transform into the continuous sine/cosine phase sequence $a[n]$ and $b[n]$, which then can be filtered by the mean filter as

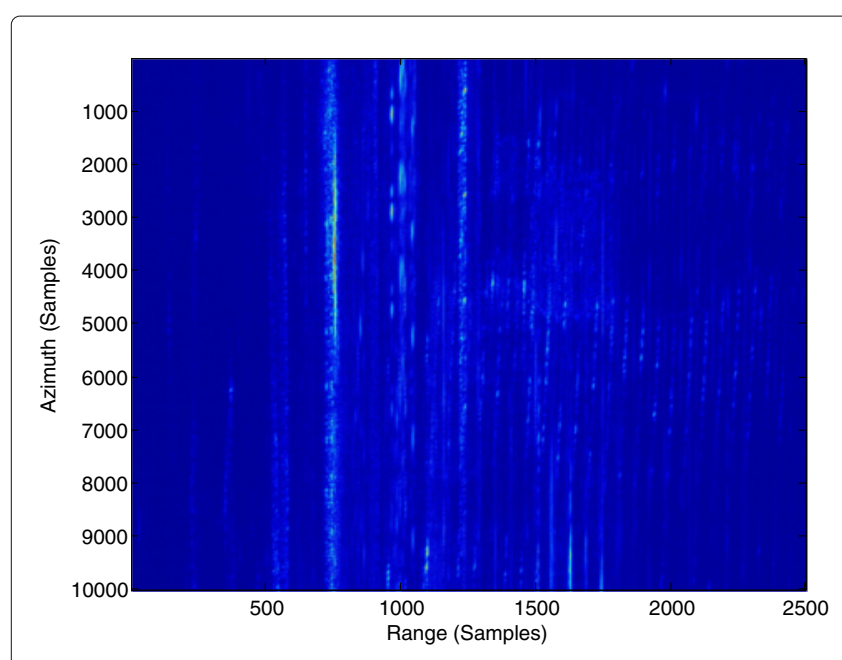

(a)

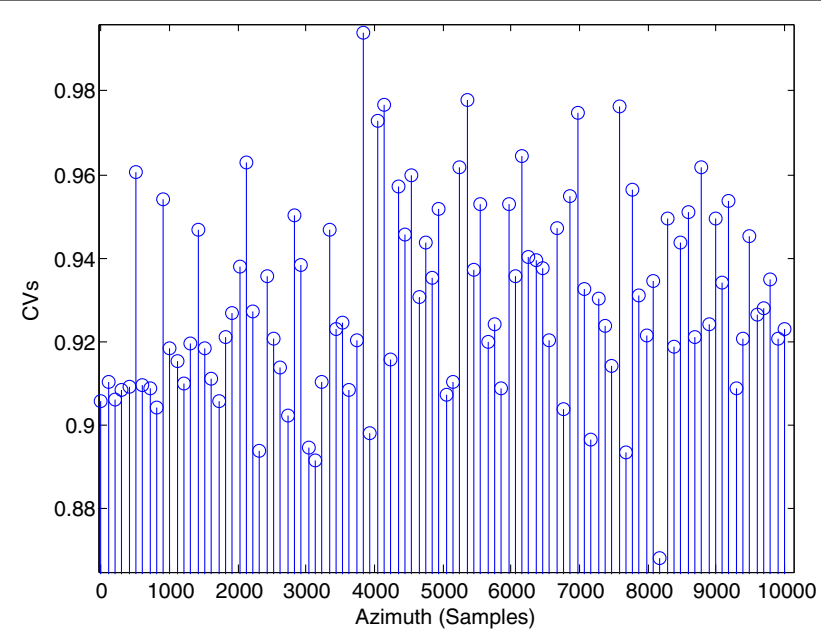

(b)

Fig. 4 a Range focused signal of the real data. $\mathbf{b}$ CV between adjacent rows 


$$
\begin{aligned}
& \bar{a}[n]=\frac{1}{M} \sum_{i \in W} a[n] \\
& \bar{b}[n]=\frac{1}{M} \sum_{i \in W} b[n]
\end{aligned}
$$

where $W$ is the neighborhood with center $n$ and $M$ is the size of mean filter window. In the phase difference curve, the jitter at some locations is large, while other locations are relatively flat. Therefore, the size of the mean filter window $\mathrm{M}$ can not be a constant value, but should change with a specific parameter. When the jitter is large, a small window should be used; otherwise, it is likely to make the curve too flat. When the jitter is flat, a larger window should be used, which makes the curve as smooth as possible. Calculate the arctangent function through the filtered $\sin /$ cosine values as

$$
\Phi_{1}[n]=\arctan \frac{\bar{b}[n]}{\bar{a}[n]}
$$

By using the arctangent function as inverse mapping, the filtered sawtooth phase is limited in the interval of $(-\pi / 2, \pi / 2]$. By judging the quadrant of the phase difference sequence through the positive and negative relationship between sine and cosine values, the filtered smooth phase difference sequence can be determined. After the phase difference denoising procedure, the jitter in the phase difference decreases a lot, which makes the phase unwrapping more accurately.

In principle, the displacement $n_{m+1}$ can be estimated by fitting the slope of the phase difference curve $\Phi_{1}[n]$. However, the displacement estimated at this point is inaccurate. The reason is that there exists some minute differences between two adjacent rows due to the noise and clutter in practical application. The corresponding solution will be discussed in the next subsection.

\subsection{Low-frequency fitting}

Information between $f_{m}\left[n-n_{m+1}\right]$ and $f_{m+1}[n]$ can be divided into macroscopic similarity and detailed difference, which correspond to low-frequency and high-frequency components in frequency spectrum, respectively. The low-frequency part, which corresponds to the similarity of adjacent azimuth cells, is a data segment similar to the ideal phase curve, and it can be fit to a straight line. While the high-frequency part, which corresponds to the detailed difference of adjacent azimuth cells, cannot be fit correctly.

Therefore, taking the low-frequency spectrum information to fit the phase difference curve with the least-squares method is a feasible way precise estimation. In the following, a strategy to select the low-frequency part is presented.

1. The first-order differential of the phase difference curve can be calculated as

$$
\Delta \Phi[n]=\Phi_{1}[n]-\Phi_{1}[n-1]
$$

2. By using a mean filter, the derivative curve is smoothed.

$$
\varphi[n]=\frac{1}{M} \sum_{i \in W} \Delta \Phi[n]
$$

where $W$ is the neighborhood with center $n$ and $M$ is the size of mean filter window.

3. Search peaks of $\varphi[n]$ from the central zero frequency. If the peak value is larger than the threshold, set the fitting area between zero and the peak value.

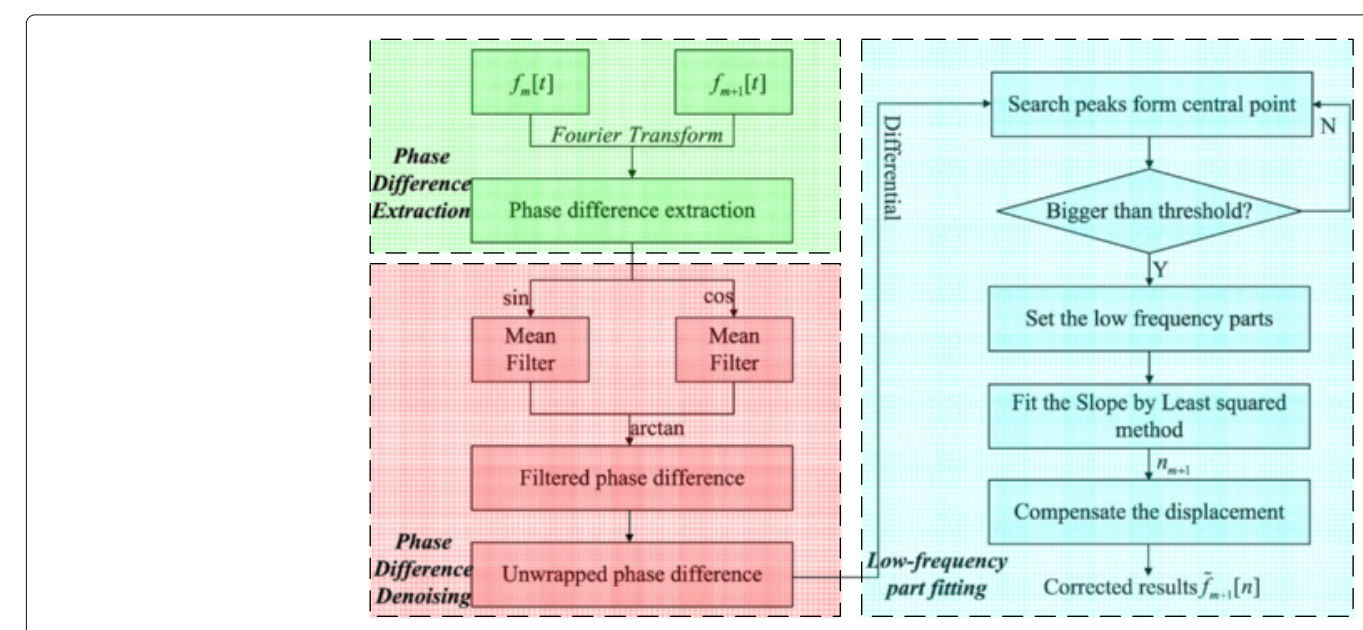

Fig. 5 Flowchart of the proposed method for a single azimuth cell 


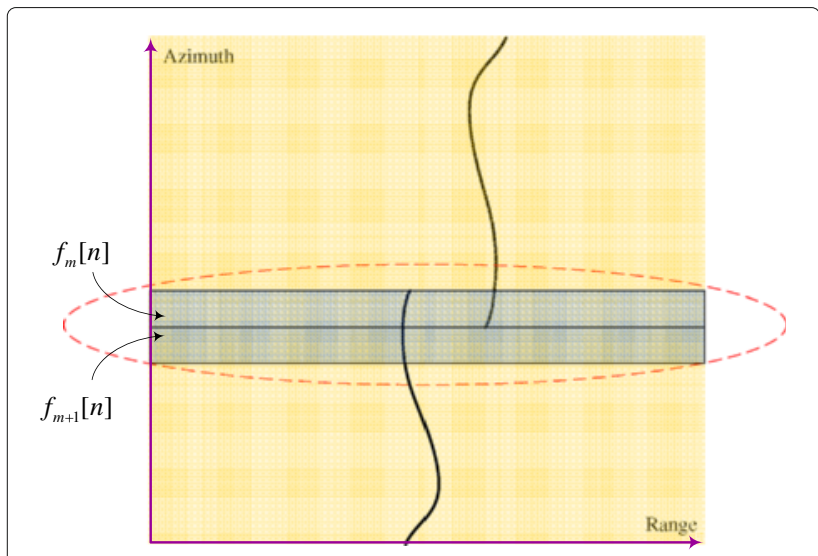

Fig. 6 Illustration of residual RCM in the presence of two dominant targets

The differential phase difference in step 2 presents a notch area in the low-frequency part. The adjacent average method in step 2 aims at reducing false positives, which means avoiding putting the jitter low-frequency points into the high-frequency points. Step 3 selects the low-frequency part by searching the notch area of differential phase difference.

At this point, least-squares method is utilized to fit the slope $n_{m+1}$ in the low-frequency part. Then, corresponding correction result $\tilde{f}_{m+1}[n]$ can be achieved according to (22).

$$
\tilde{f}_{m+1}[n]=\operatorname{IFT}\left[F_{m+1}(\omega) e^{j \omega n_{m+1}}\right]
$$

To make it clear, the flowchart of the proposed residual RCM correction method for a single azimuth cell is shown in Fig. 5.

\subsection{Discussion}

In the proposed method, a dominant point-target model, i.e., the presence of one strong scatterer per footprint.
Indeed, when only distributed targets are present significant decorrelation between adjacent azimuth cells is expected to appear. For example, when two dominant targets are located in the scene, the signal after coarse range focusing is shown in Fig. 6.

As for the azimuth bins $f_{m}[n]$ and $f_{m+1}[n]$, there are significant decorrelation, and the relationship in Eq. (9) cannot be satisfied. In this condition, the assumption for the proposed method is not valid, and we cannot estimate the range deviation difference between these two range bins. In order to cope with this problem, the $\mathrm{CV}$ between the adjacent azimuth bins should be calculated first. Then, as for the azimuth bins that the CV is smaller than the threshold, we do not estimate the range deviation difference. After the range deviation differences of most azimuth bins are estimated, the range deviations of the azimuth bin, whose $\mathrm{CV}$ is smaller than the threshold, are estimated based on the range deviation difference of the adjacent azimuth bins by the fitting methods.

In addition, computational complexity of the proposed method is analyzed here. Suppose the range and azimuth samples are $N_{r}$ and $N_{a}$, respectively. The computational complexity of the proposed residual RCM correction method for a single azimuth cell is elaborated as follows. (1) The phase difference extraction procedure uses two times Fourier transform and two times phase factor multiplication. The computational complexity is $O\left(N_{r} \log N_{r}\right)$. (2) The phase difference denoising procedure uses five times phase factor multiplication and one 1-D phase unwrap processing. The phase unwrap processing in [13] is utilized in this paper, and the computation complexity of this processing is $O\left(N_{r}\right)$. As a consequence, the computation complexity of this phase difference denoising procedure is $O\left(N_{r}\right)$. (3) The major operations of low frequency fitting procedure are three times phase factor multiplication, one time linear search, one time inverse Fourier transform, and one time linear fitting. The computational complexity is $O\left(N_{r} \log N_{r}\right)$. As a consequence,

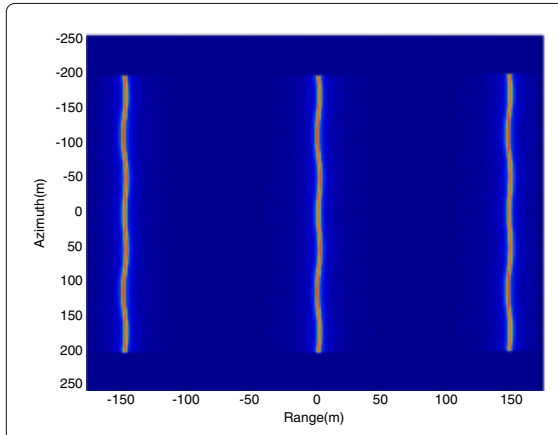

(a)

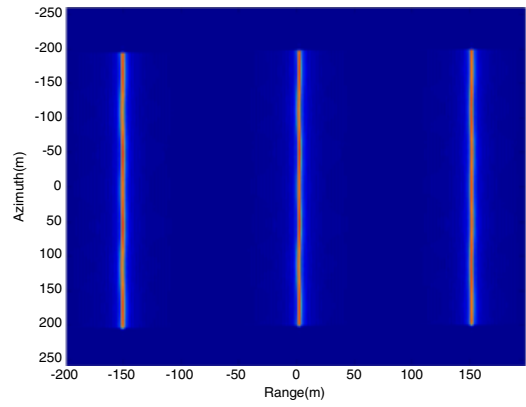

(b)

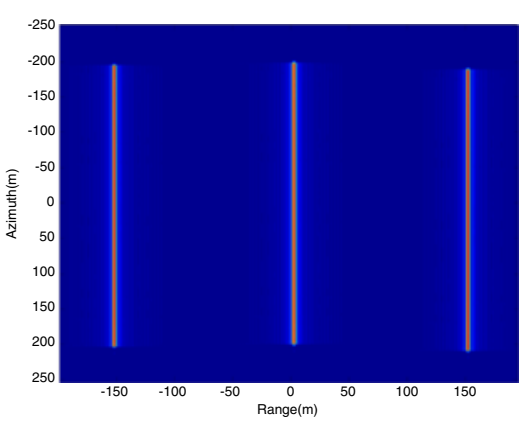

(c)

Fig. 7 a Coarse range focused data before residual RCM correction. b Results after residual RCM correction of MEM. c Results after residual RCM correction of the proposed method 


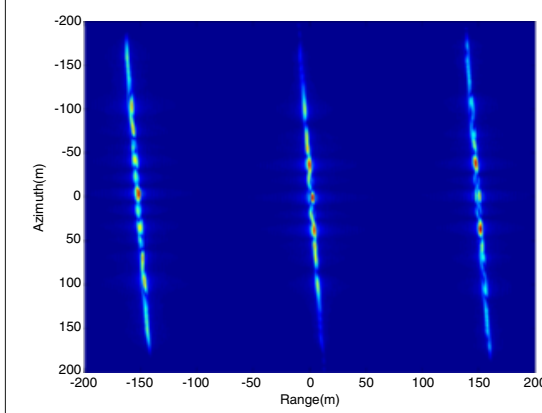

(a)

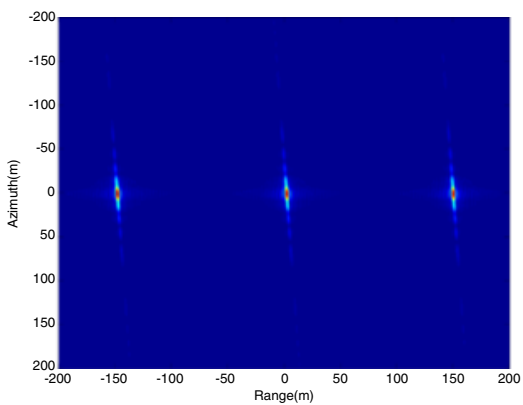

(b)

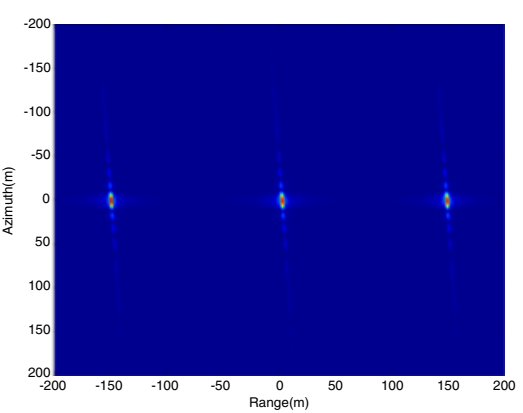

(c)

Fig. 8 a Imaging results without residual RCM correction. b Imaging results with residual RCM correction of MEM. c Imaging results with residual RCM correction of the proposed methods

the computational complexity for the whole algorithm is $O\left(N_{a} N_{r} \log N_{r}\right)$.

\section{Experimental results}

In this section, both of the point target simulation and the real data experiment are performed to evaluate the performance of the proposed residual RCM correction algorithm for BFSAR.

\subsection{Simulation and analysis}

Some simulation parameters are shown in Table 1 . Three point targets $(A, O$, and $B)$ are assumed to be distributed in the scene. The coordinates of the targets $A, O$, and $B$ are $(-200,0), 0(0,0)$, and $(200,0) \mathrm{m}$, respectively. Motion errors are added to the raw data of BFSAR. Let $\Delta x_{T}$ and $\Delta x_{R}$ denote the deviations in $x$ direction for the transmitter and receiver, $\Delta y_{T}$ and $\Delta y_{R}$ denote the deviations in $y$ direction for the transmitter and receiver, while $\Delta z_{T}$ and $\Delta z_{R}$ denote the height deviations of the transmitter and receiver, respectively. Functions of these deviations with respect to the azimuth time $t$ are presented in (23).

$$
\left\{\begin{array}{c}
\Delta x_{T}=\cos (2 \pi t), \Delta x_{R}=2 \cos (2 \pi 0.6 t) \\
\Delta y_{T}=4 \cos (1.2 \pi t)+2 \cos (\pi t) \\
\Delta y_{R}=4 \cos (1.6 \pi t)+2 \cos (7 \pi t) \\
\Delta z_{T}=2 \cos (1.8 \pi t)+\cos (\pi t) \\
\Delta z_{R}=3 \cos (7.2 \pi t)+4 \cos (\pi t)
\end{array}\right.
$$

In order to highlight the performance of this method, comparison with the minimal entropy residual RCM correction method (MEM) [6] is done. Residual RCM exists in the data after range compression and RCM correction as shown in Fig. 7a. Figure 7c denotes the corrected data by the proposed method. Compared with the nonlinear migration trajectory in Fig. 7a, the straight migration trajectory in Fig. 7c suggests that the proposed method works well to correct the residual RCM. Figure $7 \mathrm{~b}$ presents the corrected data by the residual RCM correction method in [6]. It can be seen that the proposed method has a better performance than the method in [6]. The reason is that the parametric model of the range displacement in [6] restricts the estimation accuracy.

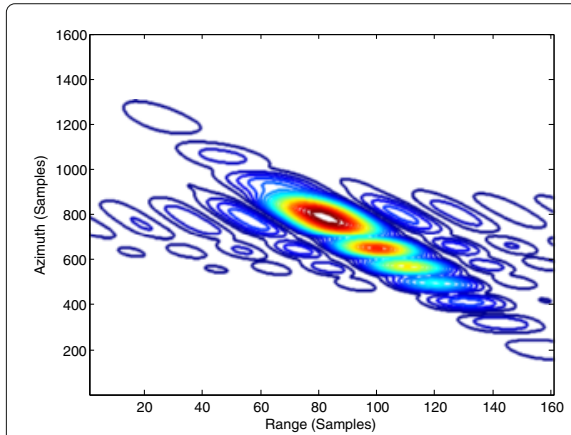

(a)

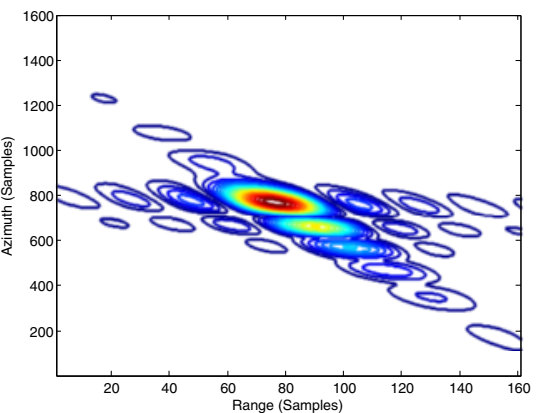

(b)

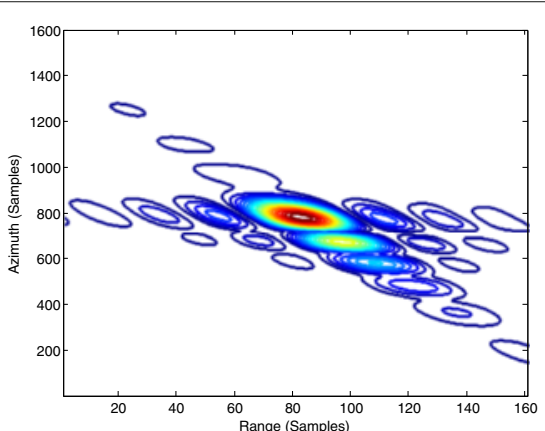

(c)

Fig. 9 Processing results by MEM. a Target $A$. b Target $O$. c Target $B$ 


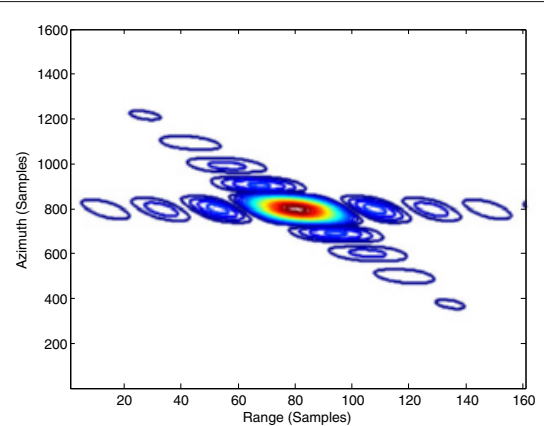

(a)

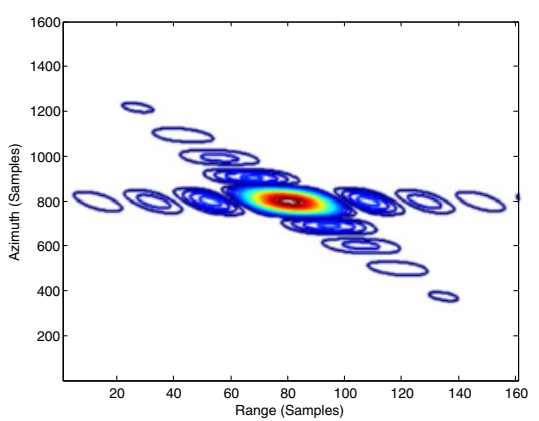

(b)

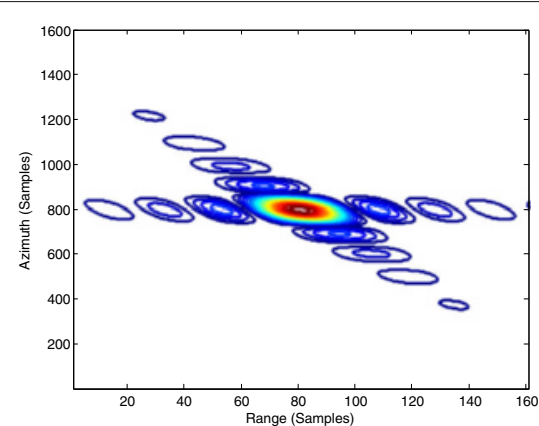

(c)

Fig. 10 Processing results by the proposed method. a Target $A$. b Target $O$. c Target $B$

After correcting the residual RCM, a robust autofocus method MIA [14] is applied to obtain the imaging results. Final compressed images of the target in the scene center are shown in Fig. 8. Quality of the image without residual RCM correction suffers considerable degradation in both azimuth and range direction in Fig. 8a. In Fig. 8b, c, the imaging qualities are improved using MEM and the proposed method.

In order to compare the performance of MEM and the proposed method, the three targets in the imaging results of MEM and the proposed method are interpolated eight times as shown in Figs. 9 and 10, respectively. The results show that we can get the perfectly focused imaging results by the proposed method, while the imaging results can not be focused well by MEM. Besides, we measure the quality of imaging results of the focused targets of the proposed method and MEM by peak sidelobe ratio (PSLR), integrated sidelobe ratio (ISLR), and impulse-response width (IRW) as shown in Table 2. From Table 2, it also can be found that the proposed method has a better performance than MEM.

True displacement and estimated displacement are shown in Fig. 11a, b, and the estimation errors are shown in Fig. 11c. It can be seen that the estimated displacement matches quite well with the true displacement in the proposed method, and the variation of the root mean square (RMS) of an overall estimation error is $0.011 \mathrm{~m}$.
Compared with the proposed method, estimation accuracy of MEM is bad, and the RMS is $1.21 \mathrm{~m}$. The reason is that the displacement estimated by MEM must be modeled as a polynomial, which restricts the estimation accuracy.

The performance of the proposed method in terms of precision and robustness to noise is evaluated. Gaussian white noise with different SNRs is added to the range compression data, and 1000 repetitions of each case were carried out. Figure 12a, b shows the RMS, calculated from simulation results, as a function of SNR by MEM and the proposed method, respectively. It is clear that if SNR is larger than $5 \mathrm{~dB}$, the RMS results obtained from the proposed method are lower than $0.012 \mathrm{~m}$. If SNR is smaller than $5 \mathrm{~dB}$, the proposed method performs poorly with SNR tending to zero. The RMS of MEM increases when the SNR becomes smaller as well. Compared with the proposed method, the estimation results show that MEM is more sensitive to noise. Future work will study the performance of the proposed method in terms of robustness to noise.

\subsection{Real data results}

In 2012, we performed an airborne BFSAR experiment which was the first airborne bistatic forward-looking experiments in the world [12]. In this experiment, the two platforms were mounted on two airplanes of Yun-5. The

Table 2 Imaging quality parameters

\begin{tabular}{|c|c|c|c|c|c|c|c|}
\hline & & \multicolumn{3}{|c|}{ Range } & \multicolumn{3}{|c|}{ Azimuth } \\
\hline & & PSLR (dB) & ISLR (dB) & IRW (m) & PSLR (dB) & ISLR (dB) & IRW (m) \\
\hline \multirow{3}{*}{$\begin{array}{l}\text { By the proposed } \\
\text { method }\end{array}$} & Target A & -13.35 & -10.22 & 0.68 & -13.19 & -10.09 & 0.88 \\
\hline & Target O & -13.37 & -10.23 & 0.67 & -13.26 & -10.17 & 0.84 \\
\hline & Target B & -13.34 & -10.22 & 0.68 & -13.14 & -10.11 & 0.86 \\
\hline \multirow{3}{*}{ By MEM } & Target A & -8.02 & -7.97 & 0.91 & -6.03 & -4.76 & 1.98 \\
\hline & Target O & -13.37 & -8.23 & 0.87 & -6.76 & -5.68 & 1.84 \\
\hline & Target B & -8.13 & -8.12 & 0.88 & -6.12 & -5.03 & 1.90 \\
\hline
\end{tabular}




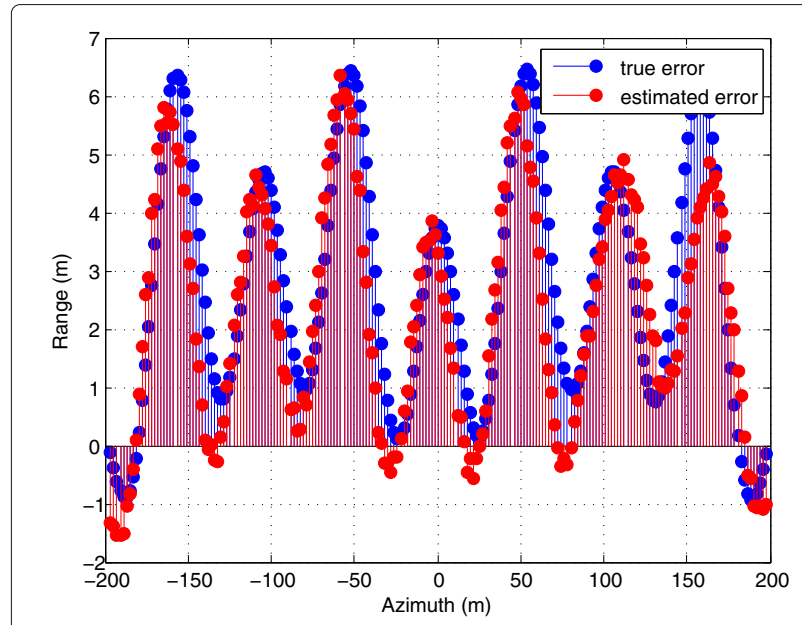

(a)

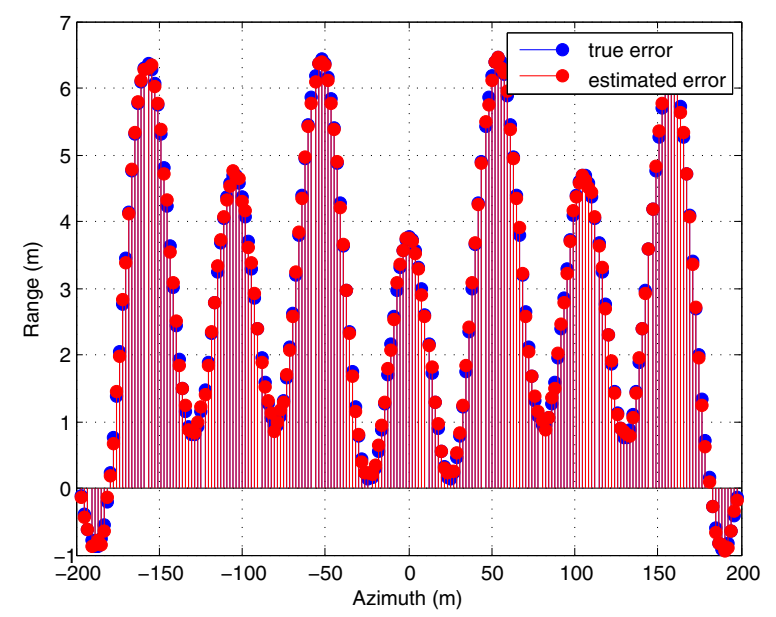

(b)

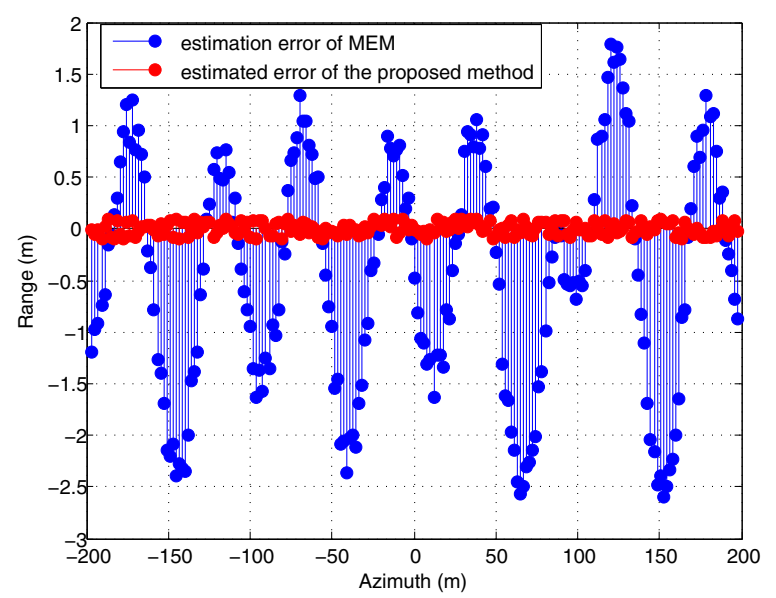

(c)

Fig. 11 a Estimation results by MEM. b Estimation results by the proposed method. c Estimation error

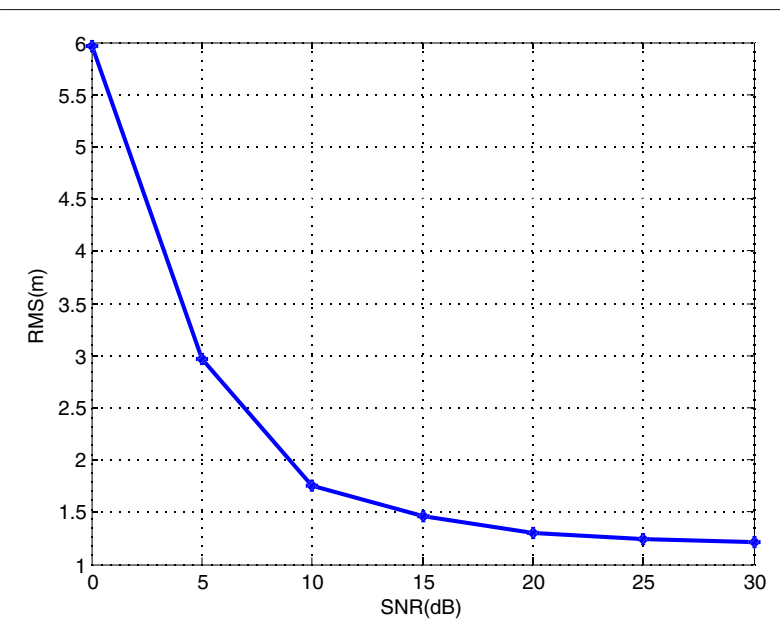

(a)

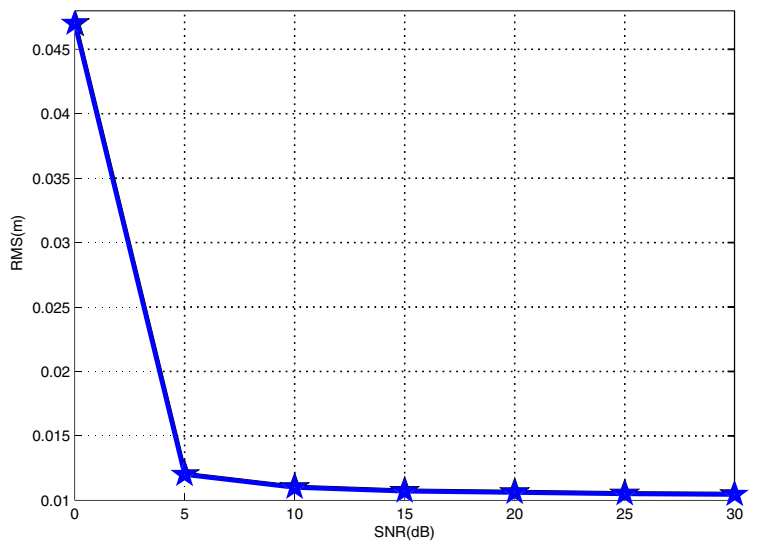

(b)

Fig. 12 a RMS of estimated residual RCM versus SNR by MEM. b RMS of estimated residual RCM versus SNR by the proposed method

antennas of the system are two horn antennas, and the overlap of the antenna footprints was guaranteed by careful flight planning and pilots skill. The system works in $\mathrm{X}$ band. The bandwidth of the transmitted chirp signal is $75 \mathrm{MHz}$. Pulse repetition frequency is $500 \mathrm{~Hz}$. The velocities of the two platforms were controlled to be $42 \mathrm{~m} / \mathrm{s}$. The geometry configuration is shown in Fig. 13.

After utilizing the proposed residual RCM correction method, MIA is applied to estimate azimuth phase errors and obtain the final imaging results, and the full compressed image is shown in Fig. 14b. Figure 14a shows the imaging results of by MIA directly without correcting the residual RCM. In Fig. 14a, b, the horizontal direction is azimuth, and the vertical direction is range. We can find that the method in this paper can obtain a little better performance than the imaging results without residual RCM correction. Reconstructions of the proposed method exhibit excellent quality. 

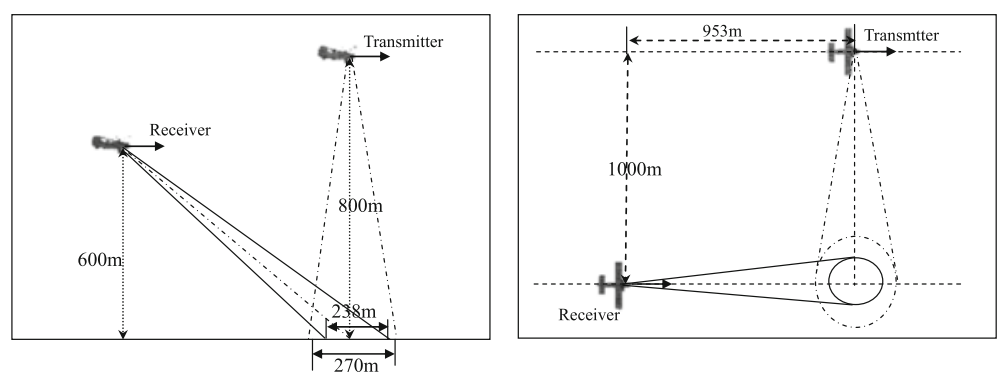

Fig. 13 Geometry configuration in the airborne experiment. a Side view. b Vertical view

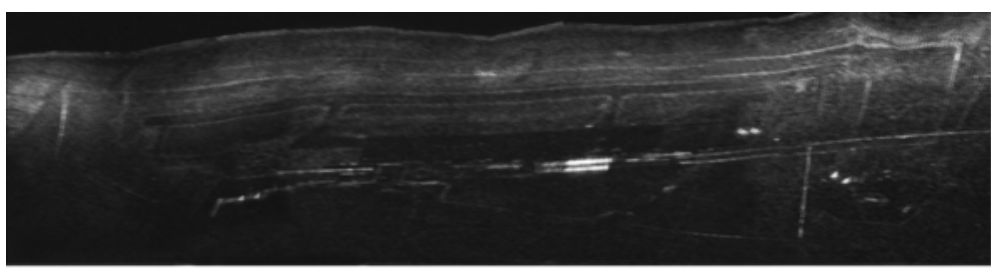

(a)

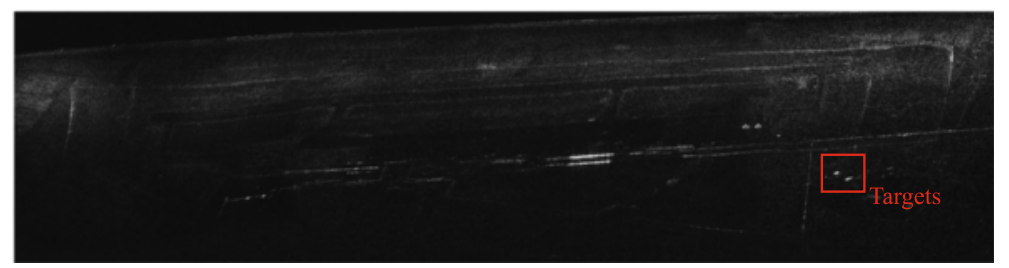

(b)

Fig. 14 a Image result without residual RCM correction. b Image result with residual RCM correction
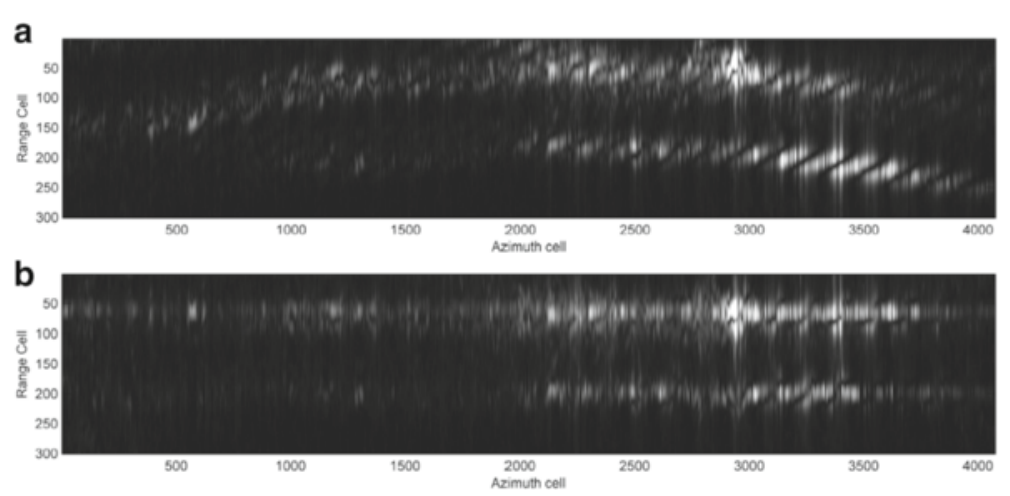

Fig. 15 a Data before residual RCM correction. b Data after residual RCM correction 
To illustrate the detail of the proposed method, migration trajectories of the targets in Fig. 14b are presented in Fig. 15. Figure 15a gives the data after range compression and LRCMC. Although deterministic range migration has been compensated by using RCM correction, residual RCM is still large enough to exceed several range resolution cells. In this situation, conventional autofocus algorithm cannot completely compensate for the errors. Figure 15b shows the imaging results utilizing MIA. It can be clearly seen that the image suffers from severely 2-D defocus.

\section{Conclusions}

In this paper, a novel residual RCM correction method based on low-frequency fitting for BFSAR has been proposed. By fitting the low-frequency phase difference between adjacent azimuth cells, residual RCM in each azimuth cell is corrected one by one. Using the leastsquares method, the estimation result is generally not an integer, and a sub-pixel level correction result can be achieved. Compared with previous works on residual RCM correction of SAR data, the proposed method can realize residual RCM correction requiring neither interpolation nor parametric model. In addition, the proposed algorithm is robust to noise. Simulations and experiments have been carried out to confirm the effectiveness of the proposed algorithm.

\section{Competing interests}

The authors declare that they have no competing interests.

Received: 15 March 2016 Accepted: 29 September 2016

Published online: 22 October 2016

\section{References}

1. X Mao, D Zhu, Y Zhang. Knowledge-aided two-dimensional autofocus for synthetic aperture radar. Radar Conference (RADAR) (IEEE, Ottawa, ON, 2013), pp. 1-6

2. $L$ Yang, M Xing, Y Wang, L Zhang, Z Bao, Compensation for the NsRCM and phase error after polar format resampling for airborne spotlight SAR raw data of high resolution.Geosci. Remote Sensing Lett. IEEE. 10, $165-169$ (2013)

3. X Mao, D Zhu, Z Zhu, Autofocus Correction of APE and residual RCM in spotlight SAR polar format imagery. Aerospace Electron Syst. IEEE Trans. 49, 2693-2706 (2013)

4. AW Doerry, Autofocus correction of excessive migration in synthetic aperture radar images, (US, Department of Energy, 2004)

5. JT González-Partida, P Almorox-González, M Burgos-García, BP Dorta-Naranjo, SAR system for UAV operation with motion error compensation beyond the resolution cell. Sensors. 8, 3384-3405 (2008)

6. J Wang, D Kasilingam, Global range alignment for ISAR.Aerospace electron. Syst. IEEE Trans. 39, 351-357 (2003)

7. G Delisle, $\mathrm{H}$ Wu, Moving target imaging and trajectory computation using ISAR. Aerospace Electron. Syst. IEEE Trans. 30, 887-899 (1994)

8. W Pu, J Yang, W Li, J Wu, Y LV, A residual range cell migration correction algorithm for SAR based on low-frequency fitting. (IEEE, Arlington, VA, 2015), pp. 1300-1304

9. W Li, J Yang, Y Huang, J Wu, A geometry-based Doppler centroid estimator for bistatic forward-looking SAR. Geosci. Remote Sensing Lett. IEEE. 9, 388-392 (2012)
10. Y Tang, B Zhang, M Xing, Z Bao, L Guo, The space-variant phase-error matching map-drift algorithm for highly squinted SAR. Geosci. Remote Sensing Lett. IEEE. 10, 845-849 (2013)

11. J Wu, Z Li, Y Huang, J Yang, H Yang, QH Liu, Focusing bistatic forward-looking SAR with stationary transmitter based on keystone transform and nonlinear chirp scaling. Geosci. Remote Sensing Lett. IEEE. 11, 148-152 (2014)

12. J Yang, Y Huang, H Yang, J Wu, W Li, Z Li, X Yang. A first experiment of airborne bistatic forward-looking SAR preliminary results. Remote Sensing Symposium (IGARSS) 2013 IEEE International (IEEE, Vic., Australia, 2013), pp. 4202-4204

13. J Tribolet, A new phase unwrapping algorithm.Acoustics Speech Signal Process. IEEE Trans. 25, 170-177 (1977)

14. TJ Kragh. Monotonic iterative algorithm for minimum-entropy autofocus. Adaptive Sensor Array Processing (ASAP) Workshop, (Lexington, MA, June 2006)

\section{Submit your manuscript to a SpringerOpen ${ }^{\circ}$ journal and benefit from:}

- Convenient online submission

- Rigorous peer review

- Immediate publication on acceptance

- Open access: articles freely available online

- High visibility within the field

- Retaining the copyright to your article

Submit your next manuscript at $>$ springeropen.com 